\title{
Individual differences in error tolerance in humans: Neurophysiological evidences
}

\author{
Gonçalo Padrao $^{1,2}$ - Aida Mallorquí ${ }^{3}$ David Cucurell ${ }^{1,2}$. $^{2}$ \\ Antoni Rodriguez-Fornells ${ }^{1,2,4}$
}

Published online: 28 May 2015

(C) Psychonomic Society, Inc. 2015

\begin{abstract}
When interacting in error-prone environments, humans display different tolerances to changing their decisions when faced with erroneous feedback information. Here, we investigated whether these individual differences in error tolerance (ET) were reflected in neurophysiological mechanisms indexing specific motivational states related to feedback monitoring. To explore differences in ET, we examined the performance of 80 participants in a probabilistic reversal-learning task. We then compared eventrelated brain responses (ERPs) of two extreme groups of participants (High ET and Low ET), which showed radical differences in their propensity to maintain newly learned rules after receiving spurious negative feedback. We observed that High ET participants showed reduced anticipatory activity prior to the presentation of incoming feedback, informing them of the correctness of their performance. This was evidenced by measuring the amplitude of the stimulus-preceding negativity (SPN), an ERP component indexing attention and motivational engagement of incoming informative feedback. Postfeedback processing
\end{abstract}

Antoni Rodriguez-Fornells

arfornells@gmail.com

1 Cognition and Brain Plasticity Group [Bellvitge Biomedical Research Institute-] IDIBELL, L'Hospitalet de Llobregat, Barcelona 08097, Spain

2 Department of Basic Psychology, Campus Bellvitge, University of Barcelona, L'Hospitalet de Llobregat, Barcelona 08097, Spain

3 Sant Pere Claver Health Foundation, Mental Health Services, Barcelona 08004, Spain

4 Catalan Institution for Research and Advanced Studies, ICREA, Barcelona, Spain
ERP components (the so-called Feedback-Related Negativity and the P300) also showed reduced amplitude in this group (High ET). The general decreased responsiveness of the High ET group to external feedback suggests a higher proneness to favor internal(rule)-based strategies, reducing attention to external cues and the consequent impact of negative evaluations on decision making. We believe that the present findings support the existence of specific cognitive and motivational processes underlying individual differences on error-tolerance among humans, contributing to the ongoing research focused on understanding the mental processes behind human fallibility in error-prone scenarios.

Keywords Error tolerance $\cdot$ Decision-making · Action-monitoring $\cdot$ ERPs $\cdot$ Individual differences

Humans have different tolerance to negative feedback about their performance and changing their ongoing behaviors based on external environmental cues. As these individual differences are likely to influence the way their decisions are acted upon, an important challenge for cognitive psychologists and neuroscientists is not only to understand the nature of the brain mechanisms and inherent cognitive and motivational processes underlying these differences but also to explain how these differences are related to (in)adequate decisional processes carried out by human agents in their interaction with real-world and error-prone environments.

The study of human error processing has advanced enormously during the last 2 decades, mostly due to the discovery of specific event-related brain components (ERPs) and neural networks that respond selectively to error monitoring, error correction, and compensation processes (Carter et al., 1998; 
Gehring, Goss, Coles, Meyer, \& Donchin, 1993; Holroyd \& Coles, 2002; Marco-Pallares, Camara, Munte, \& RodriguezFornells, 2008; Ridderinkhof, Ullsperger, Crone, \& Nieuwenhuis, 2004; Rodriguez-Fornells, Kurzbuch, \& Munte, 2002; for a review, see Ullsperger, Danielmeier, \& Jocham, 2014). However, most of this research has been focused exclusively on the examination of isolated performance errors (i.e., slips of action or lapses) in fairly simple reaction time tasks, whereby errors are carried out without conscious deliberation (Norman, 1981; Reason, 1990) and are mostly due to small attentional fluctuations or maladjustments in control (Botvinick, Braver, Barch, Carter, \& Cohen, 2001; Gratton, Coles, Sirebaag, Eriksen, \& Donchin, 1988). However, there is a clear lack of neurophysiological research focused on the more complex error-prone scenarios or related to individual differences in on-line monitoring of complex chains of commands (Norman, 1988; Rasmussen, 1983; Reason, 1990; Woods, 1994). It is also worth noting that many catastrophes are often related to errors in human decision-making processes during the operation of complex technological systems (e.g., drivers, air traffic controllers; Casey, 2006; Wiegmann \& Shappell, 2003; Woods, 1994). Thus, it is important to move this research forward and to further investigate other factors that contribute to human failures in these settings (Reason, 1990). An attractive alternative is, for instance, to explore how individual differences in error tolerance (ET) may influence human decision processes and the extent to which these differences are explained by discrete neurophysiological mechanisms related to the monitoring and evaluation of error feedback in more uncertain and error-prone environments.

The concept of error tolerance (ET) in complex systems makes reference to the robustness of a system to continue functioning in accordance with design specifications even when undesired or unpredictable changes in the environment occur (Albert, Jeong, \& Barabasi, 2000). This concept has been important in the design of computer modeling of operating systems (Fields, Wright, \& Harrison, 1995) and even in understanding the degree of tolerance against errors in simple living organisms (Jeong, Tombor, Albert, Oltvai, \& Barabasi, 2001). In human performance, error-tolerance has been investigated in low-level sensoriomotor learning, in which a large degree of noise, variability, and redundancy exist in movement execution (Jeong et al., 2001; Sternad, Abe, Hu, \& Muller, 2011) as well as in speech motor control (Houde \& Nagarajan, 2011).

Adapted to decision making, the study of ET could be particularly relevant in better characterizing the cognitive and motivational processes underlying the way humans cope with errors and multiple feedback information in hazardous environments. We understand, in the present research, the concept of ET as the tendency or bias of human agents, in the presence of conflicting or error feedback information, to follow welllearned behaviors or ongoing action plans (e.g., well-known routines) without the need to drastically change preselected courses of action. For instance, in more uncertain and errorful environments, individuals with higher ET may be more prone to exploit well-known routines or behavioral schemes that might have been successful in previous situations, showing an increased resistance to new errorful feedback information. Accordingly, these individuals might operate predominantly in a feedback-independent fashion, favoring internal(rule)-based strategies and directing less attention to external cues. In turn, individuals with lower ET may be less resistant to negative feedback and thus more likely to change their ongoing behavior and to explore new alternatives after negative evaluations. Moreover, due to their cognitive schemas, individuals with high or low ET levels may naturally incur in different types of mistakes or diagnostic errors (Reason, 1990), which certainly compromises their effectiveness during complex decisionmaking processes requiring the monitoring of different types of negative feedback information.

In this sense, individual differences in ET among humans may reflect distinctive cognitive schemas and motivational processes of self-reinforcement (Bandura, 1989) in order to cope with errors and negative feedback information. Drawing on this idea, in the present study we aimed to investigate two extreme groups of participants (with High and Low ET) in order to examine whether their strategies to cope with negative feedback during rule-based decision-making processes would be associated with different type ERP signatures that index the motivational significance of negative feedback information.

An interesting type of higher order errors in the study of ET in human performance are those described by Reason (1990; see also Rasmussen, 1983), the so-called rule-based mistakes. Rule-based mistakes usually take place when the operator intentionally formulates a plan and carries it out using inappropriate rules (Norman, 1988; Reason, 1990). In this case, the agent might correctly identify the current goal but, through lack of knowledge, strength of the habit, effort, or motivational disengagement, applies an inappropriate rule.

An experimental paradigm particularly suited to assess differences in ET is the reversal learning (RL) task (see. e.g., Jocham, Neumann, Klein, Danielmeier, \& Ullsperger, 2009). In the RL task, participants have to monitor different types of negative error feedback in order to learn stimulus-response associations that lead to rewarding outcomes. Critically, participants must adapt their behavior according to unpredicted changes in stimulus-response contingencies, that is, their decisions based on the trade-off of their rule-based knowledge (i.e., the pattern of responses awarded up to a particular moment) and the feedback received from the environment. In some cases this feedback can be randomly misleading (spurious feedback) — not rewarding the correct action or signaling the need for a behavioral change. Here, we examined the performance of 80 healthy participants during the RL task in order to select two extreme groups of individuals that showed radical differences in their propensity to maintain newly 
learned rules in spite of receiving different types of error feedback. Participants who perseverated with the newly learned rule for longer periods of time, independently of receiving error feedback, were assigned to the High ET group. In contrast, those participants who exhibited a higher tendency to change their behavior after receiving negative feedback were assigned to the Low ET group.

To assess ERP differences regarding the motivational impact of negative feedback on performance between these two ET groups we studied the activity of the feedback-related negativity (FRN) and the P3 component. The FRN is a frontocentral negative component, which arises 250 to $350 \mathrm{~ms}$ after sensory feedback. The FRN has been consistently associated with a performance-monitoring system that rapidly evaluates the motivational significance of ongoing events, being especially sensitive to negative feedback information and violations of expectancy (Gehring \& Willoughby, 2002; Holroyd \& Coles, 2002; Nieuwenhuis, Holroyd, Mol, \& Coles, 2004; Yeung, Holroyd, \& Cohen, 2005). In turn, the P3 is a slow centro-parietal component associated with the updating of relevant (i.e., motivationally salient) external information, formation of new decisions, and consequent changes in behavior according to task contingencies (Barcelo, Escera, Corral, \& Perianez, 2006; Chase, Swainson, Durham, Benham, \& Cools, 2011; Donchin \& Coles, 1988; Fischer \& Ullsperger, 2013). For instance, the $P 3$ component is usually enhanced after stimuli indicating the request to implement a new rule change (e.g., in the Wisconsin Card Sorting Task and the Reversal Learning Task; Chase et al., 2011; Cunillera et al., 2012). Centro-parietal EEG activity indexed by the P3-like components may possibly echo brain computations, reflecting the accumulation of internal evidences that an error (or erratic behavioral trend) has occurred (Steinhauser \& Yeung, 2010) and deliberate changes in behavior may be required. Recent studies have suggested that centro-parietal EEG activity following relevant feedbacks (the P3) and salient errors (the Pe) reflect similar neural computations involved in the conscious processing of motivationally significant events (Ridderinkhof, Ramautar, \& Wijnen, 2009; Steinhauser \& Yeung, 2010) and perhaps deliberate shifts in strategy (Ullsperger, Fischer, Nigburg, \& Endrass, 2014).

Furthermore, we were interested in measuring differences in the degree of participant expectation or anticipation toward external feedback using the stimulus preceding negativity (SPN). The SPN is an ERP component that is built up during the period preceding feedback presentation and has been described as an electrophysiological marker of subjects' anticipatory attention and motivational engagement to informative or relevant feedback (Brunia, Hackley, van Boxtel, Kotani, \& Ohgami, 2011; Fuentemilla et al., 2013; Kotani et al., 2003; Masaki, Takeuchi, Gehring, Takasawa, \& Yamasaki, 2006; Moris, Luque, \& Rodriguez-Fornells, 2013).
Based on the assumption that individuals with High ET and Low ET, tend to favor different strategies to cope with negative feedback, we expected to observe different types of rulebased mistakes between the two groups as well as ERP differences regarding the motivational engagement during error feedback anticipation (indexed by the amplitude of the SPN component) and error feedback evaluation (indexed by amplitude of the FRN and P3 components). Specifically, we expected to observe an increased tolerance (resistance) to negative feedback information in the High ET group when compared to the Low ET group, reflected by a decreased activity of the SPN, FRN and P3 components.

\section{Method}

\section{Participants}

Forty right-handed participants who underwent the ERPreversal learning task were selected among 80 subjects from the University of Barcelona. Participants were divided in two groups (20 subjects each) based on their performance in the RL task (see the categorization of High and Low ET groups). Both groups did not differ statistically in either age- - High ET: $22 \pm 4$; Low ET: $21 \pm 4, t(38)=0.4, p>.05$ - or gender, $\chi^{2}(1$, 40) $=1.2, p>.05)$.

Participants reported no history of neurological or psychiatric disorder or drug consumption. All participants signed an informed consent and received monetary compensation that varied in accordance to their performance accuracy, $23.3 \pm 2.1$ $€($ mean $+S D)$. The data from these participants has not been presented elsewhere.

\section{Psychometric assessment}

All participants were screened with questionnaires designed to assess personality measures related to aversive and approach motivation and responsiveness to rewards and punishments. We selected the Sensitivity to Punishment and Sensitivity to Reward Questionnaire (SPSRQ; Torrubia, Ávila, Moltó, \& Caseras, 2001) and the BIS/BAS subscales (Carver \& White, 1994). The SPSRQ is composed of two scales: the Sensitivity to Punishment scale (SP) and the Sensitivity to Reward scale (SR). The SP items assess behavioral responses to situations involving novelty or aversive consequences, as well as cognitive processes produced by the threat of failure in goals. SR items describe situations in which people employ efforts to obtain rewarding experiences (e.g., money, sex partners, or social events). Regarding the BIS/BAS scales, the Behavioural Inhibition System subscale (BIS) is sensitive to cues that anticipate punishment and trigger negative affect towards potentially stressful events. In turn, the Behavioural Approach System (BAS) is subdivided in three subscales that 
assess proneness to engage in goal-directed efforts and positive affect in response to impending reward cues: (1) the BAS Drive subscale evaluates persistence to chase desired goals; (2) the items on the Fun Seeking (FS) subscale evaluate desire and willingness to approach a potentially rewarding event; and (3) the Reward Responsiveness (RR) subscale is focus on positive responses to the occurrence or anticipation of rewarding events.

\section{Experimental design}

Participants performed a probabilistic reversal learning task. The task consisted of 1,260 trials divided in 63 blocks, with 16 to 24 trials each (pseudorandom order). In each trial, two gray squares located on either side of a central fixation point were presented over a black background for 1,000 ms. Participants had to select one of the two squares by pressing one of two mouse buttons that corresponded to each stimulus. $700 \mathrm{~ms}$ after the response (delay period-feedback anticipation), a feedback stimulus indicating a win or loss of $0.06 €$ (feedback processing: happy or sad face, respectively) was presented in the middle of the screen for $800 \mathrm{~ms}$ (see Fig. 1A). Intertrial stimulus was set to 500 to

A

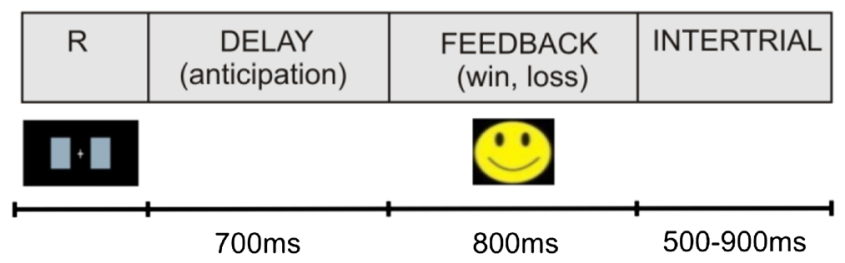

B

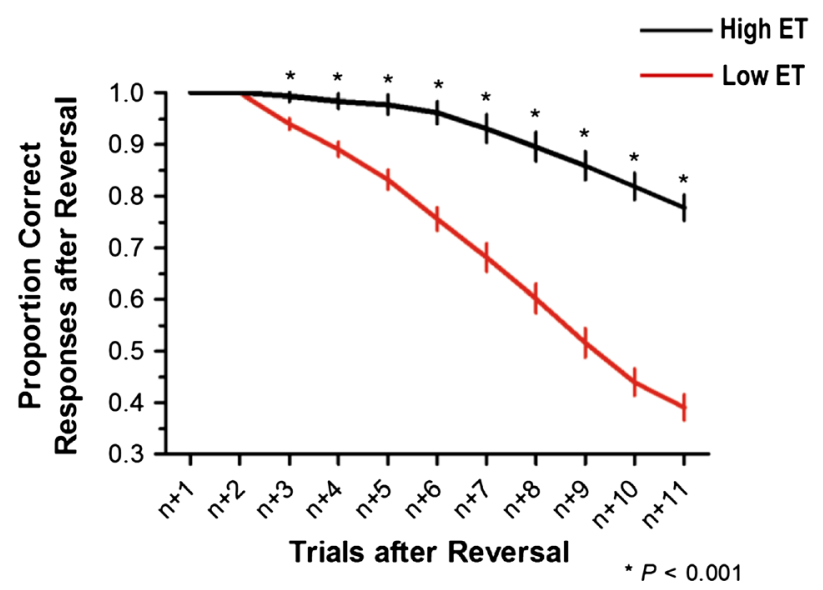

Fig. 1 A. Schematic illustration of the task design. For each trial, participants had to choose one of the squares placed on each side of the screen by pressing the corresponding mouse button. After a delay period of $700 \mathrm{~ms}$, feedback indicating a monetary win or loss was presented. B. Perseveration of the rule in High (black) and Low (red) ET groups. The $x$-axis shows the number of trials after a successful reversal of behavior (i.e., immediately following the Final reversal error). The values on the $y$ axis are the percentage across 11 trials (mean $+S E M)$ in which the participants maintained the current rule
$900 \mathrm{~ms}$ (randomized) to avoid automatic responses. When participants did not respond within $1,000 \mathrm{~ms}$ after the presentation of the two squares, a question mark appeared in the screen. Selfpaced resting breaks for free blinking were given every three blocks coinciding with the 7th through 10th trial of each block to avoid any participants' anticipation of the rule change. Information about the amount of money gained until that moment was provided during these breaks.

Participants were informed of the existence of two probabilities of winning and losing (75\% and $25 \%$, respectively) associated with each stimulus. By trial and error, subjects had to learn which stimulus was the more rewarded. Critically, after a randomly jittered block length of 16 to 24 trials, stimulus-reward contingencies were reversed (rule reversal), and participants had to switch their selection to the new rewarded alternative. Thus, due to the $75 \%$ probability of winning, participants had to monitor different types of negative (error) feedback, which in some cases could be misleading, nonrewarding the correct response according to the ongoing rule (referred to here as spurious negative feedback), or signaling the need of a behavioral change (when concurring with the rule reversal, referred to here as reversal trials; see Table 1). Importantly, participants were explicitly instructed to start choosing the other stimulus alternative only when they were completely sure that the rule had changed.

A brief training session was conducted at the beginning of the session to ensure comprehension of the task. Participants were encouraged to increase their earnings as much as possible during the task.

\section{Selection of the participants based on ET}

Fifteen participants from the 80 that performed the RL task were excluded from analysis following data collection due to excessive noise in the EEG signal (see the Method section). From the remaining 65 participants we selected two extreme groups (each composed of 20 participants), which at the behavioral level showed radical differences regarding their propensity to keep on with learned rules even when receiving negative feedback information. According to the characteristics of the $\mathrm{RL}$ task, ET was defined as the propensity of a participant to maintain a learned rule after a successful reversal despite the presence of misleading or spurious error feedback (note that only $75 \%$ of correct trials were rewarded). Therefore, to select the members of each group we inspected participants' performance throughout the 11 consecutive trials following a successful behavioral switch (i.e., when the rule has actually reversed and participants had to switch to the new correct response; see Fig. 1B); Specifically, we plotted the data of all 65 participants showing the proportion of trials after reversal, in which the new learned rule was maintained throughout the 11 consecutive trials (averaged across all 62 experimental blocks). The most extreme participants ( $n=20$ per group) who persevered with the 
Table 1 An example of a sequence of trials in the probabilistic reversal learning task, including the categorization of the trials according to the subject's response and the feedback obtained. In this particular sequence of trials, the first rule (A: press Left for reward) was maintained during 14 trials. After the last trial, it changed to the opposite rule (B: press Right for obtaining reward). For a definition of each category see the Method section

\begin{tabular}{|c|c|c|c|c|}
\hline Rule & $\begin{array}{l}\text { Rewarded } \\
\text { response }\end{array}$ & $\begin{array}{l}\text { Subject's } \\
\text { response }\end{array}$ & Event type & Feedback \\
\hline A & Left & Left & Reward & (:) \\
\hline A & Left & Left & Reward & (:) \\
\hline A & Left & Left & Reward & (:) \\
\hline A & Right & Left & $\begin{array}{l}\text { SPURIOUS } \\
\text { NEGATIVE }\end{array}$ & $\odot$ \\
\hline A & Left & Left & Reward & (:) \\
\hline A & Left & Left & Reward & (:) \\
\hline A & Left & Left & Reward & (:) \\
\hline A & Right & Left & $\begin{array}{l}\text { SPURIOUS } \\
\text { NEGATIVE }\end{array}$ & $\odot$ \\
\hline A & Left & Right & $\begin{array}{l}\text { LOSS-SHIFT } \\
\text { ERROR }\end{array}$ & $\odot$ \\
\hline A & Left & Left & Reward & (:) \\
\hline A & Left & Left & Reward & (:) \\
\hline A & Left & Left & Reward & (:) \\
\hline A & Left & Left & Reward & (:) \\
\hline A & Left & Left & Reward & (:) \\
\hline $\begin{array}{l}\text { RULE } \\
\text { change B }\end{array}$ & Right & Left & $\begin{array}{l}\text { REVERSAL } \\
\text { TRIAL }\end{array}$ & $\odot$ \\
\hline B & Right & Left & $\begin{array}{l}\text { REVERSAL } \\
\text { ERROR }\end{array}$ & $\odot$ \\
\hline B & Right & Left & $\begin{array}{l}\text { FINAL } \\
\text { REVERSAL } \\
\text { ERROR }\end{array}$ & $\odot$ \\
\hline B & Right & Right & Reward & (:) \\
\hline B & Right & Right & Reward & (:) \\
\hline
\end{tabular}

new learned rule for longer periods of time, independently of the presence of spurious (error) feedback or error feedback informing of a rule change, were assigned to the High ET group. In contrast, the 20 participants that exhibited more problems in maintaining the new learned rule, swinging between the response alternatives more often after receiving negative feedback, were assigned to the Low ET group (see Fig. 1B). Because we were unable to collect the psychometric data from four participants (two participants from each of the ET groups), the final sample for the behavioral and ERP analysis included 18 subjects in each group.

\section{EEG recording}

EEG activity was recorded using tin electrodes located at 29 standard positions $(\mathrm{Fp} 1 / 2, \mathrm{Fz}, \mathrm{F} 7 / 8, \mathrm{~F} 3 / 4, \mathrm{Fc} 1 / 2 \mathrm{Fc} 5 / 6, \mathrm{Cz}$, C3/4, T3/4, Cp1/2, Cp5/6, Pz, P3/4, T5/6, PO1/2, O1/2) mounted in an elastic cap. Vertical eye movements were monitored with an electrode at the infraorbital ridge of the right eye. Electrode impedances were kept below $5 \mathrm{k} \Omega$. The electrophysiological signals were digitized at a rate of $250 \mathrm{~Hz}$ and filtered with an online band pass of 0.01 to $70 \mathrm{~Hz}$ (half-amplitude cut-offs). All scalp electrodes were referenced offline to the mean activity of the left and right mastoids.

\section{Behavioral and ERP analysis}

Different types of events were defined for the behavioral and ERP analysis of the RL task (see Table 1 for clarification):

1. Spurious negative feedback (SN). An unexpected (misleading) negative (error) feedback (monetary loss) received by the participant after a correct rule response, due to the $25 \%$ probability of receiving an error or incorrect feedback. Importantly, we included only those trials that were preceded by at least five positive feedback trials, which indicate that the current rule was correctly consolidated for that particular block.

2. Reversal trial (RT). The first negative error feedback concurring with a rule shift and a new starting block.

3. Final reversal error (FRE). The last negative error feedback after which the participant switched his or her response pattern to the correct new rule.

4. Positive (POS) feedback (rewarded) trials. Rewarded responses after a correct response that followed the expected rule. For the ERP analysis, only those trials that followed the fifth trial after the rule change were selected to ensure that the new rule has been correctly consolidated.

The reaction times (RTs) from these types of trials were also analyzed to inspect possible differences between the two groups. The mean RTs were submitted to repeated measures ANOVAs with factors error feedback type (Positive-correct, Spurious negative, Reversal trial, and Final reversal error) and Group (Low ET and High ET), as a between-subject factor.

Regarding the behavioral performance we also inspected the following events:

1. Reversal errors. Perseverative errors after a reversal trial. These errors (see Table 1) occurred when the participant did not change the response pattern after the rule changed (reversal trial).

2. Loss-shift errors. After receiving an error feedback that was not consistent with a rule shift (spurious negative feedback), the participant changed the response incurring a monetary loss.

\section{ERP analysis}

EEG data was low-pass filtered offline at $12 \mathrm{~Hz}$ for the ERP analysis. ERPs related to anticipation/expectation toward the 
outcome (the SPN component) were studied by extracting response-locked EEG epochs of $900 \mathrm{~ms}$ starting $100 \mathrm{~ms}$ before the button press (baseline). ERP components associated with negative (error) feedback processing/evaluation (FRN and $\mathrm{P} 3$ components) were investigated by epoching EEG data from $-100 \mathrm{~ms}$ (baseline) to $600 \mathrm{~ms}$, time locked to the feedback onset. Trials from the first block were not entered into the analysis. Trials exceeding $\pm 100 \mu \mathrm{V}$ in both EEG and EOG were excluded from further analysis.

The time windows for the statistical analyses of ERPs were chosen based on visual inspection of the grand average waveforms.

Changes in the SPN amplitude were studied in the 200-ms period prior to feedback presentation, which, according to previous studies, reflects the temporal window of maximal SPN activity (Masaki et al., 2006; Moris et al., 2013).

The FRN amplitude in response to error feedback was calculated as the difference waveform negative (error) feedback minus positive (reward) feedback trials for each of the conditions of interest (Spurious negative, Reversal trial, Final reversal error) within a time window located $60 \mathrm{~ms}$ around the peak of activity (280-340 ms). Performing the analysis on the difference waveform allowed us to (a) isolate the FRN signal that developed superimposed onto a slow positive waveform (the P3), (b) stabilize possible drifts, and (c) control differences between the two groups regarding general feedback processing on reward-correct trials. Changes in the P3 amplitude in response to negative feedback were also examined on the difference waveform between negative (error) feedback and positive (reward) feedback trials by averaging amplitudes within a window of 350 to $500 \mathrm{~ms}$.

Anticipatory processes (SPN) toward negative feedback information were examined with ANOVAs with two withinsubjects factors: error feedback type (Spurious negative, Reversal trial, Final reversal error) and electrode location (Fz, $\mathrm{Cz}, \mathrm{Pz}$ ), and one between-subject factor Group (Low ET and High ET). The analysis of different types of negative feedback was important in assessing differences in error tolerance regarding neural expectation processes towards redundant/misleading (Spurious negative events) and relevant learning error feedback information (Reversal trial and Final reversal error). The same method was applied to test statistical effects on the mean amplitude of the FRN (280-240 ms) and P3 (350-500 ms) components during error feedback evaluation. These two neural responses directly indicated the motivational impact of error feedback processing in different error-tolerant systems.

Furthermore, differences between the two groups regarding neural processes related to anticipation and evaluation of positive feedback (rewarded trials), co-occurring with correct rule-based responses, were also tested using repeated measures ANOVAs using factors of electrode location (frontal, Fz; central, Cz; parietal, Pz) and Group (Low ET and High ET) on the mean amplitude of the SPN (anticipation) and P3 (evaluation). These analysis assessed differences between the two groups regarding general feedback processing (i.e., participants' tolerance to external cues informing about correct performance). The two groups did not differ regarding the number of analyzed EEG trials in each of the conditions of interest: Positive feedback, High ET: $247 \pm$ 10; Low ET: $237 \pm$ $30, t(34)<1$; Spurious negative, High ET: $134 \pm 10$; Low ET: $135 \pm 10, t(34)<1$; Reversal trials, High ET: $50 \pm 4$; Low ET: $52 \pm 6, t(34)=1.5, p>.05$, and Final reversal errors, High ET: $42 \pm 8$; Low ET: $39 \pm 6, t(34)=1.1, p>.05$.

\section{Results}

\section{Psychometric data}

Psychometric information from the two ET groups is summarized in Table 2. No significant differences were observed between both ET groups in any of the scales evaluated (SP, SR, BIS and Bas).

\section{Behavioral performance}

Participants were divided into two groups based on their propensity to sustain the new rule after task contingencies changed and independently of the presence of spurious negative feedbacks (i.e., a distinctive feature of High ET group). To examine the differences in the behavioral patterns of both groups, we first analyzed the average of participants' correct responses (across all 62 blocks) throughout 11 trials $(n+11)$ after the rule change. This measure gave us an index of the proportion of trials in which participants were likely to maintain the current learned rule across time. A repeated measures ANOVA was carried out including Trial number (from 1 to 11 after correct rule change) and Group (High ET and Low ET). A main effect of Trial number showed a clear overall decrease in the tendency to maintain the new rule across time, $F(10,340)=233.9, p<.0001, \eta p^{2}=$ 0.87 (Fig. 1B). The significant interaction Trial number $x$ Group,

Table 2 Mean scores $( \pm S D)$ of the High and Low ET groups on the SPSR subscales (sensitivity to punishment, SP; sensitivity to reward, SR) and BIS and BAS subscales (drive, reward responsiveness, RR; fun seeking, $\mathrm{FN}$ ) as well as independent $t$ test comparisons between groups $(n s, p>.05)$

\begin{tabular}{llllll}
\hline Scales & High ET & Low ET & $t$ test & $p$ value & Cohen's $d$ \\
\hline$S P$ & $13(6)$ & $13(7)$ & 0.26 & $n s$ & 0.009 \\
$S R$ & $8(3)$ & $10(4)$ & 1.9 & $n s$ & 0.65 \\
$B I S$ & $23(3)$ & $21(6)$ & 1.5 & $n s$ & 0.51 \\
$B A S$ drive & $12(2)$ & $12(2)$ & 0.6 & $n s$ & 0.20 \\
$F S$ & $12(2)$ & $11(3)$ & 0.9 & $n s$ & 0.30 \\
$R R$ & $16(2)$ & $16(3)$ & 0.3 & $n s$ & 0.12 \\
\hline
\end{tabular}


$F(10,340)=58.4, p<.0001, \eta p^{2}=0.63$, showed that while the Low ET group exhibited a higher tendency to switch between the two response alternatives more frequently, the High ET group showed a greater tolerance to error feedback and therefore "stuck to" the learned rule for extended periods of times. The two groups began to significantly differ from each other from the third trial after the reversal (pairwise comparisons $t$ test at all-time points, $p<.001$ ).

Compared to the High ET group, the Low ET showed a higher amount of Loss-shift errors, Low ET: $36 \pm 23$, High ET: $5 \pm 4: t(34)=5.6, p<.001, d=1.9$. These mistakes demonstrate the tendency of the Low ET group to change their response pattern right after receiving negative feedback, even when the rule had not changed. In contrast, the proportion of Reversal errors was greater in the High ET group, High ET: $140 \pm 18$; Low ET: $96 \pm 14: t(34)=8.7, p<.001, d=2.9$. On average, the High ET group needed more error feedbacks to change the current rule after a Reversal trial, High ET: $3.2 \pm$ 0.35; Low ET: $2.3 \pm 0.27: t(34)=7.2, p<.001, d=2.4$. These results clearly showed that the two groups of ET were differently influenced by negative feedback information. On one hand, High ET participants seem to be less influenced by negative feedback, being more prone to follow the learned rule for extended periods of time, with the cost of committing a higher number of perseverative errors and taking more time to adapt behavior after reversal compared to the Low ET group. On the other hand, Low ET participants were more likely to change ongoing correct decisions after negative feedback, incurring in a higher number of mistakes related to an inadequate monitoring of spurious feedback information (i.e., Lost-shift errors). It is worth mentioning that the rigidity of the strategies adopted by each one of the two ET groups may have compromised the overall effectiveness on the task, as the amount of money collected along the task did not differ between them (High ET: $23.1 \pm 2.4 €$; Low ET: $23.1 \pm 1.9 €, p>.5$ ).

Furthermore we inspect possible differences in RTs between the two groups during Positive-correct, Spurious negative, Reversal trials, and Final reversal errors. Although the High ET group was in general faster responding than the Low ET group ( $275 \mathrm{~ms} \pm 45$ vs. $300 \mathrm{~ms} \pm 71$ ), the differences between the two groups did not reach statistical significance, $F(1,34)=3.4, p=.074, \eta p^{2}=0.091$. No differences were observed between the different type of trials, $F(3,102)=2.4, p$ $>.05, \eta p^{2}=0.067$, and the interaction feedback type $\mathrm{x}$ Group was not significant, $F(3,102<1$.

\section{ERP results}

\section{Anticipation and processing of positive feedback}

To evaluate differences in ET related to the anticipation and processing of expected positive outcomes that signaled the current (rewarded) rule selection, we investigated the amplitude of the SPN and P3 components (see the summary in Table 3). Statistical effects on the SPN mean amplitude (200 ms period preceding feedback presentation) were evaluated with an ANOVA, including Electrode location (Fz, Cz, $\mathrm{Pz}$ ) and Group (Low ET and High ET; see Fig. 2A). A main effect of Group was encountered, $F(1,34)=7.5, p<.05, \eta \mathrm{p}^{2}=$ 0.18 , pointing to reduced SPN amplitude in the High ET group (see Fig. 2A). This group difference was maximal at location $\mathrm{Cz}$, electrode location $\mathrm{x}$ Group interaction, $F(1,34)=$ $6.6, p<.05, \eta p^{2}=0.162$; see the scalp distribution of the SPN component for the difference Low ET-High ET in Fig. 2A)

Complementing the previous results, a clear decrease was also observed in the P3 amplitude (350-500 ms) for the High ET group, main effect of Group, $F(1,34)=13.8, p<.001, \eta^{2}$ $=0.29$; see Fig. $2 \mathrm{~B}$ ) after the presentation of the positive feedback. The differences between both groups were larger at $\mathrm{Pz}$, electrode location $\mathrm{x}$ Group interaction, $F(2,68)=3.9$, $p<.05, \eta \mathrm{p}^{2}=0.1$; see the centro-parietal distribution of the $\mathrm{P} 3$ in Fig. 2B). This reduction in the amplitude of the $\mathrm{P} 3$ was expected considering the reduced motivational impact that expected feedback might have on participants' whose decisions are internal-rule based (i.e., High ET group).

A correlation analysis (Pearson coefficient) between the amplitude of the SPN and the amplitude of the P3 further showed that greater SPN activity was associated with greater P3 enhancements $(r=-0.6, p<.001$, computed at Pz location). This correlation was still present when both groups were analyzed separately: High ET $(r=-0.5, p=.035)$; Low ET ( $r$ $=-0.57, p=.014$ ). This association observed is in agreement with the idea that the larger the expectancy towards external feedback, the larger the motivational impact of its presentation (Fuentemilla et al., 2013; Moris et al., 2013).

\section{Anticipation of negative error feedback: SPN}

To inspect differences in the motivational engagement of the participants before receiving relevant or spurious error feedback, we first examined the modulation of the SPN component in response to different types of error feedback during reversal. An ANOVA analysis was conducted with factors error feedback type (Spurious negative, Reversal trial, Final reversal error), electrode location ( $\mathrm{Fz}, \mathrm{Cz}, \mathrm{Pz}$ ), and Group (Low ET and High ET; see the summary in Table 3).

The High ET group exhibited an overall reduction of the SPN compared to the Low ET group, main effect of Group, $F(1,34)=4.9, p<.05, \eta p^{2}=0.13$; see Figs. $3 \mathrm{~A}$ and $\left.5 \mathrm{~A}\right)$. The differences between groups were higher at $\mathrm{Cz}$, quadratic model electrode location $\mathrm{x}$ Group interaction, $F(1,34)=9.4, p<$ $.005, \eta p^{2}=0.22$; see in Fig. 4A the scalp distribution of the SPN for the difference between both groups. The SPN amplitude was modulated by the relevance of the feedback, main effect of error feedback type, $F(2,68)=28.5, p<.001, \eta p^{2}=$ 0.46 , being greater (i.e., more negative) just before Final 
Table 3 Summary of the mean amplitude $(\mu \mathrm{V} \pm S E M)$ of the SPN $(\mathrm{Cz}$ electrode), FRN (Fz electrode) and P3 (Pz electrode) components in the High and Low ET groups during different type of negative error feedback: SN, spurious negative; RT, reversal trial; FRE, final reversal trial; POS, positive feedback; ..., the FRN was not analysed in POS trials

\begin{tabular}{lllll}
\hline & SN & RT & FRE & POS \\
\hline SPN & High ET: $3.97 \pm 0.7$ & High ET: $3.96 \pm 0.7$ & High ET: $0.28 \pm 0.7$ & High ET: $3.93 \pm 0.7$ \\
$(\mathrm{Cz})$ & Low ET: $1.02 \pm 0.6$ & Low ET: $0.61 \pm 0.7$ & Low ET: $-1.11 \pm 1.0$ & Low ET: $0.93 \pm 0.7$ \\
FRN & High ET: $-0.17 \pm 0.8$ & High ET: $1.22 \pm 0.4$ & High ET: $5.11 \pm 0.9$ & High ET: $\ldots$ \\
$(\mathrm{Fz})$ & Low ET: $-2.91 \pm 0.8$ & Low ET: $-1.39 \pm 1.0$ & Low ET: $2.65 \pm 1.0$ & Low ET: $\ldots$ \\
P3 & High ET: $3.78 \pm 0.7$ & High ET: $5.57 \pm 0.8$ & High ET: $9.58 \pm 0.7$ & High ET: $1.98 \pm 0.8$ \\
$(\mathrm{Pz})$ & Low ET: $6.69 \pm 1.5$ & Low ET: $7.51 \pm 1.4$ & Low ET: $8.79 \pm 1.2$ & Low ET: $6.4 \pm 0.8$ \\
\hline
\end{tabular}

reversal errors (FRE), that is, the last error feedback preceding correct behavioral shifts, when compared to Spurious negative, $t(35)=5.4, p<.001, d=0.8$, and Reversal trials, $t(35)=$ $4.8, p<.001, d=0.76$; see Fig. $5 \mathrm{~A}$ ). The increase of the SPN during Final reversal errors is important because it signals the moment of updating of the current rule and, naturally, the period in which participants may generate greater expectancies and direct more attention to the incoming feedback. A marginal interaction of error feedback type x Group, $F(2$, $68)=2.9, p=.065, \eta p^{2}=0.79$, further showed that although the SPN amplitude differed between the two groups during Spurious negative, independent sample $t$ tests at $\mathrm{Cz}, t(34)=$ $3.15, p<.005, d=1.1$, and Reversal trials, $t(34)=3.5, p<$ $.005, d=1.2$, the enhancement of the SPN amplitude just before the presentation of the error feedback preceding correct behavioral changes was similar in both groups, Final reversal errors, independent sample $t$ tests at $\mathrm{Cz}, t(34)=1.1, p>.05, d$ $=0.40$; see Fig. 5A).

Furthermore, because the differences between the two groups seem to emerge as earlier 500 to $400 \mathrm{~ms}$ prior to feedback presentation (see Fig. 3A), we conducted an additional analysis on the SPN mean voltages during two distinct time windows (400-200 ms and 200-0 ms prior to feedback presentation). The ANOVAs with factors error feedback type $\mathrm{x}$ electrode location $\mathrm{x}$ time window (400-200 ms; 200-0 ms) x Group (High ET and Low ET) confirmed that the High ET group exhibited an overall reduction of the SPN compared to the Low ET group, $F(1,34)=4.7, p<.05, \eta p^{2}=0.12$. The SPN amplitude was modulated by the relevance of the feedback, main effect of error feedback type, $F(2,68)=24.6, p<$ $.001, \eta p^{2}=0.42$, being greater during Final reversal errors (FRE) when compared to Spurious negative and Reversal trials. In agreement with previous studies (Masaki et al., 2006; Moris et al., 2013), the SPN increase during motivationally engaging and relevant feedback (in this case, Final reversal errors) was greater during the time window of 200 to $0 \mathrm{~ms}$ preceding feedback onset as revealed by the interaction error feedback type $\mathrm{x}$ time, $F(2,68)=11.2, p<.001, \eta \mathrm{p}^{2}=0.25$. A triple interaction error feedback type $\mathrm{x}$ time $\mathrm{x}$ Group, $F(2,68)$ $=4.6, p<.05, \eta p^{2}=0.12$, further showed that during the $200 \mathrm{~ms}$ preceding feedback presentation the enhancement of the SPN amplitude during Final reversal errors was stronger on the High ET group.

\section{Processing of negative error feedback: FRN and P3}

Neurophysiological processes associated with the evaluation of negative error feedback (the FRN and P3 signals) were
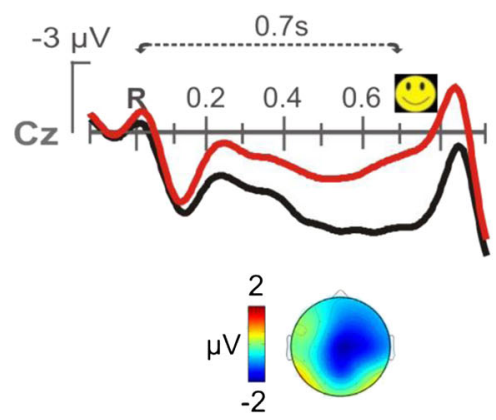

Fig. 2 ERPs regarding A, anticipation (SPN modulation at electrode $\mathrm{Cz}$ ) and $\mathbf{B}$, evaluation (P3 modulation at electrode Pz) of positive feedback (rewards). Note the increase of the SPN and P3 components on the Low ET group. Also depicted are the topographical maps of the SPN (A, time
B

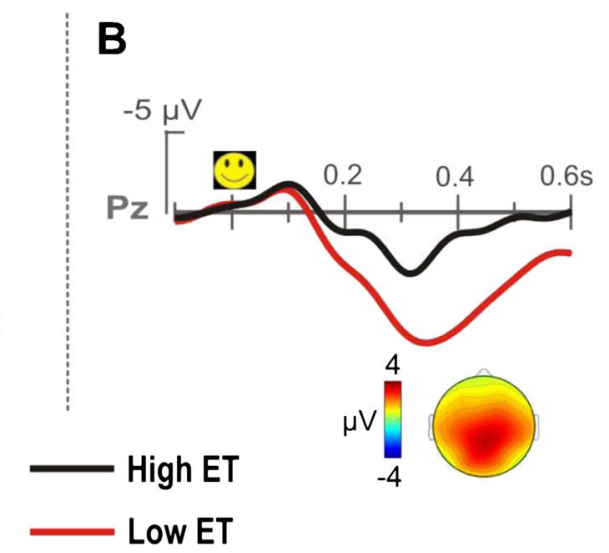

window of $200 \mathrm{~ms}$ preceding feedback presentation) and P3 components (B, time window of 350-500 ms) for the difference between Low ET minus High ET 
A
Spurious Negative
Reversal Trial
Final Reversal Error
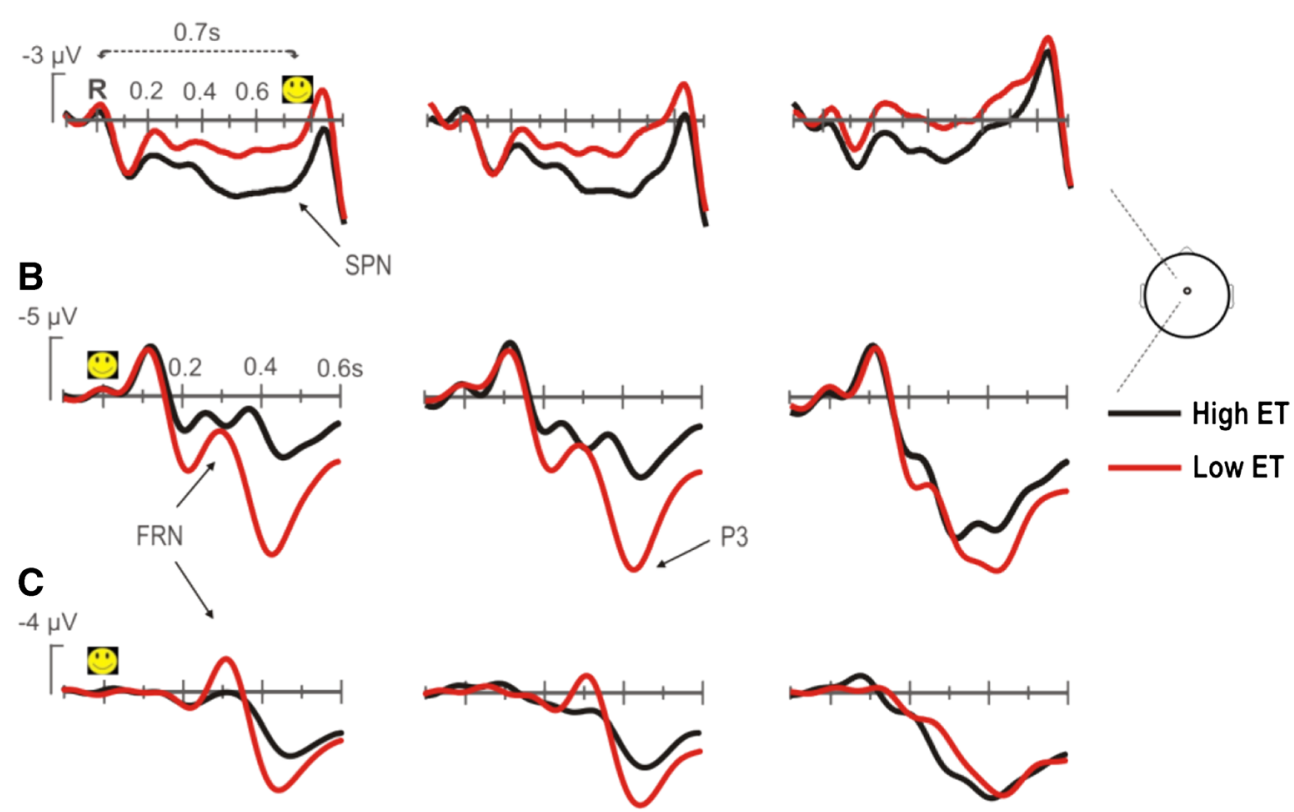

Fig. 3 A. Grand-average waveforms at electrode $\mathrm{Cz}$ during the anticipation of different type of negative error feedback in High (black) and Low (red) ET groups. Note the development of the SPN component prior to feedback presentation (feedback anticipation). B. Grand-average waveforms at electrode $\mathrm{Cz}$ in High and Low ET groups after the

inspected using ANOVAs with factors error feedback type (Spurious negative, Reversal trial, Final reversal error) $\mathrm{x}$ electrode location (Fz, Cz, Pz) x Group (Low ET and High ET).

The FRN component, calculated as the difference waveform (negative-positive feedback), showed a fronto-central distribution with maximal activity at electrode $\mathrm{Fz}$, main effect of electrode, $F(2,68)=15.9, p<.0001, \eta p^{2}=0.32$; see the classic fronto-central distribution of the FRN during the different type of negative error feedback in Fig. 4B). The amplitude of the FRN was modulated by the type of error feedback, $F(2,68)=62.4, p<.0001, \eta p^{2}=0.64$; feedback type $\mathrm{x}$ electrode, $F(4,136)=9.7, p<.001, \eta p^{2}=0.22$; the FRN amplitude was greater during unexpected negative outcomes (Spurious negative) and, conversely, expected negative feedback that preceded rule-based behavioral changes (Final reversal error) were not accompanied by an FRN. Paired $t$ tests confirm the increase of the FRN at the Fz electrode in Spurious negative compared to Reversal trials, $t(35)=4.5, p$ $<.001, d=0.45$, and Final reversal errors, $t(35)=6.8, p<$ $.001, d=1.5$, and a significant difference between Reversal trials and Final reversal errors, $t(35)=6.8, p<.001, d=1.0$. Overall, the amplitude of the FRN was lower in the High ET group when compared to the Low ET group, main effect of Group: $F(1,34)=10.6, p<.005, \eta p^{2}=0.24$, confirming the predictions that subjects with High ET are in general less responsive to error feedback (see Figs. 3C,4B, and 5B). The presentation of negative error feedback (feedback evaluation). Note the development of the FRN and P3 components during the processing of negative feedback. C. Difference waveform between negative feedback and positive (reward) trials for each of the conditions of interest $(\mathrm{Cz}$ electrode)

differences between the two groups remain stable across the different type of error feedback trials as shown by a nonsignificant interaction error feedback x Group, $F(2,68)<1$; see Fig. 5B).

The P3 component showed maximal activity over centroparietal locations ( $\mathrm{Cz}$ and $\mathrm{Pz}$ electrodes), $F(2,68)=17.7, p<$ $.001, \eta p^{2}=0.34$; see Fig. 4 C). Centro-parietal P3 activity was influenced by the type of error feedback, $F(2,68)=12.7, p<$ $.001, \eta \mathrm{p}^{2}=0.24$; error feedback x electrode, $F(4,136)=10.3$, $p<.001, \eta p^{2}=0.23$, with larger activity after Final reversal errors when compared to Reversal trials, paired $t$ test at $\mathrm{Pz}$ electrode, $t(35)=3.9, p<.001, d=0.6$; and Spurious negative, paired $t$ test at $\mathrm{Pz}$ electrode, $t(35)=5.0, p<.001, d=$ 0.85; see Figs. 3C, $4 \mathrm{C}$, and $5 \mathrm{C}$ ). An interaction error feedback type $\mathrm{x}$ Group interaction, $F(2,68)=4.1, p<.05, \eta p^{2}=0.23$, also indicated that the $\mathrm{P} 3$ enhancement after the negative feedback preceding behavioral changes compared to spurious error feedback was more abrupt on the High ET group (see Fig. 5C). No main effect of Group was seen for the P3 signal, $F(1,34)=1.3, p>.05, \eta p^{2}=0.04$.

\section{Discussion}

In the present study, we investigated whether individual differences in ET were predictive of discrete neurophysiological 
A

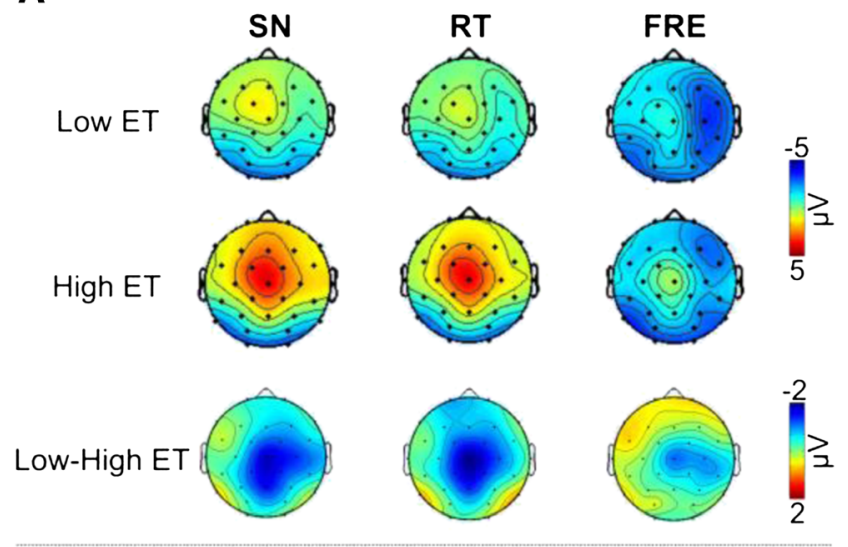

B
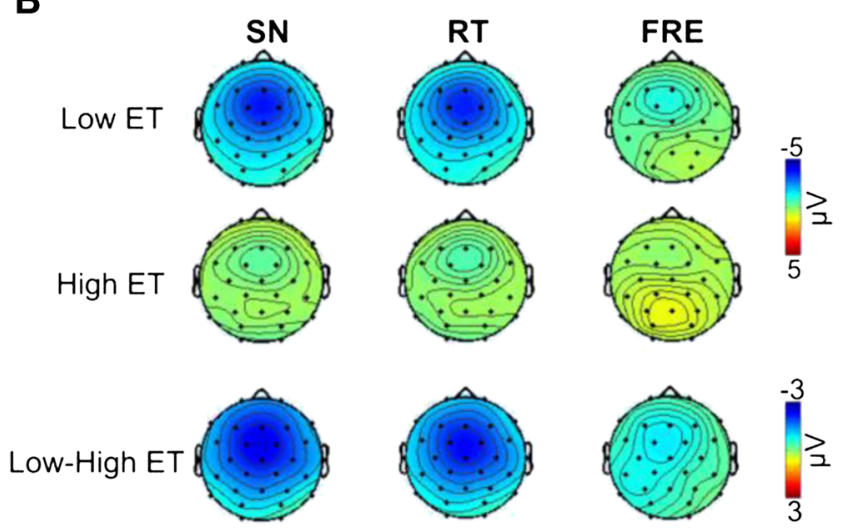

C
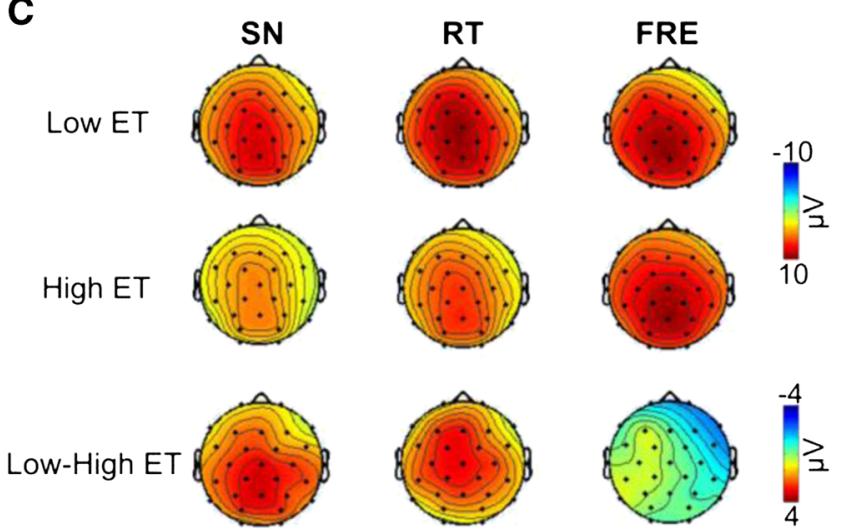

Fig. 4 Topographical maps of the ERP activity during the anticipation (SPN) and evaluation (FRN and P3) of negative error feedback (SN, spurious negative; RT, reversal trial; FRE, final reversal error) in Low and High ET groups and the difference Low ET minus High ET. A. Scalp topography of the SPN (average signal over the $200 \mathrm{~ms}$ prior to feedback presentation); B. FRN (280-340 ms); and C. P3 (350-500 ms)

mechanisms reflecting motivational brain states underlying the monitoring of error feedback presented in dynamic environments involving complex rule-base decisions. Using a reversal learning task, we compared the neurophysiological (ERPs) responses of two extreme groups of participants with High and Low ET. These two groups were selected based on their different propensity to perseverate (or change) on learned response patterns (i.e. rule-based learning) in spite of the presence of error feedback information, which could be either misleading (Spurious negative feedback), conflicting with ongoing correct conduct, or implying the reprograming of new rules and adaptive changes in behavior. It is worth mentioning that all participants were explicitly instructed to change their behavior only when they were certain that a received error feedback was concomitant with rule changes.

Despite the given instructions, we observed that during each learning block there was one cluster of the participants that was more prone to maintain the learned rule for longer periods of time, whereas another group of participants tended to switch between the response alternatives more frequently (see Fig. 1B). Individuals categorized as High ET after learning a new rule were more averse to change their behavior, displaying a higher tolerance to new incoming negative feedback, keeping on with the learned rule for longer periods of time even when the feedback was informative that the current rule was no longer valid. High ET was associated with an increased incidence of perseverative errors (i.e., reversal errors). According to Rasmussen and Vicente (1989), perseverative errors echo the application of inappropriate expectations or inefficient monitoring of environmental cues, probably due to the strength of the habit or motivational disengagement. The prevalence of perseverative errors on the High ET group reflects a cognitive bias (cognitive conservatorism or changeaversion) to favor internal-rule-based commands rather than external (environmental) guided information, devaluing new incoming information and exploiting behaviors that have been successful in previous situations (e.g., well-learned routines). In this sense, this group displayed a strategy that could be characterized as feedback independent in which the trial-bytrial feedback information has a lesser influence on subsequent decisions. In contrast, participants assigned to the Low ET group changed their ongoing behavior more regularly, especially after receiving negative feedback. After negative feedback, the Low ET group incurred in a larger number of Lost-shift errors (i.e., mistakes caused by inadequate change of the current rule), displaying a tendency to explore more regularly if fast behavioral switches after negative feedback could provide positive consequences.

Interestingly, both groups ended the task with equal gains. This finding suggests that different thresholds of ET in humans are associated with different decision-making strategies, which still can lead to similar final outcomes, being both equally advantageous and disadvantageous. In this sense, ET seems to be an important construct in understanding trial-bytrial variations in decision-making patterns and individual differences in the propensity to make different types of mistakes in uncertain and fast-changing environments.

To better illustrate this relationship between ET and (in)adequate decisional processes in complex real-life environments, let us consider the situation of an operator with 

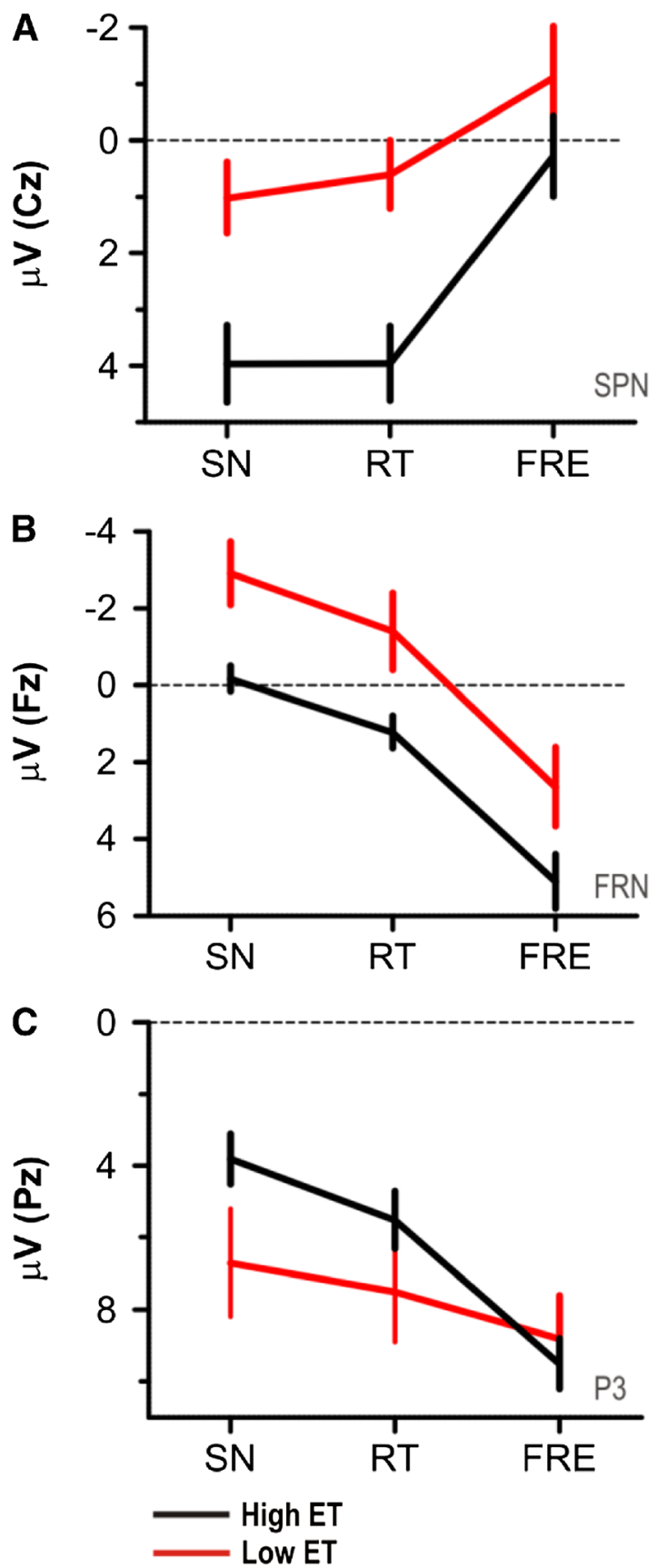

Fig. 5 A. Mean amplitude of the SPN in High (black) and Low (red) ET groups during the anticipation of negative error feedback (SN, spurious negative; RT, reversal trial; FRE, final reversal error) at electrode Cz. B. Mean amplitude of the FRN (280-340 ms) at electrode Fz during the evaluation of negative error feedback (SN, RT and FRE). C. Mean amplitude of the P3 (350-500 ms) at electrode Pz during the evaluation of negative error feedback (SN, RT and FRE). Error bars represent SEM

High ET in a flight simulator. Here the operator must handle multiple auditory and visual alarms and displays, informing him or her of the current functioning and status of the machine or of potential risks and issues requiring solutions. We suggest that individuals with High ET are more conservative and tenacious in uncertain environments in which multiple feedback needs to be constantly monitored; that is, they tend to follow well-known instructions and give little attention to external informative cue feedback. This rigid approach has its own advantages and inconveniences. On one hand, it may prevent inadequate changes in correct procedures in the face of misleading information, as reflected by a small number of Lossshift errors in this group. On the other hand, this tendency may be accompanied by less cognitive flexibility to recognize important cues demanding new behaviors, compromising them to react quickly to potential "threats" and to potentially commit perseverative mistakes more often (Cañas, Quesada, Antoli, \& Fajardo, 2003; Norman \& Shallice, 1986; Rougier, Noelle, Braver, Cohen, \& O'Relly, 2005; Spiro, Coulson, Feltovich, \& Andersib, 1988). The other way around might be the case for Low ET subjects who, being faster in reacting to cues demanding rule-based adaptive changes, are more prone to change ongoing correct actions following spurious information. We believe these behavioral differences expose distinctive cognitive schemas and motivational processes of self-reinforcement to deal with uncertainty. It is worth mention, though, that these differences cannot be explained by aspects related to aversive and approach motivation to external cues indexing punishments or rewards, as the two groups did not showed significant differences in the psychometric measures related to these aspects of motivation (the SPSPQ and the BIS/BAS scales). Subjects with High ET compared to subjects with Low ET do not necessarily tend to avoid more frequently situations involving negative evaluations or are less engaged in tasks involving negative feedback; rather, they tend to be less engaged by and direct less attention to external feedback-related information, as compared to Low ET individuals.

Looking at the neurophysiological mechanisms underlying error feedback monitoring, it was shown that participants with High ET when compared to those with Low ET exhibited a general reduction of the SPN (see Fig. 3A), FRN, and P3 (see Fig. 3B) activity during the anticipation and processing of error feedback that was not directly associated to rule-based behavioral changes (i.e., Spurious negative and Reversal trials). High ET participants also showed reduced amplitude of the SPN and P3 components on positive feedback that reinforced the current rule application (see Fig. 2). The diminished SPN amplitude in the High ET group suggests less attention orientation and motivational engagement towards the information conveyed by external events that did not imply rulebased changes in behavior (Brunia et al., 2011; Kotani et al., 2003; Moris et al., 2013). Furthermore, the reduced activity of the FRN and P3 components in the High ET group shows that this group may perceive error feedback as motivationally less salient or informative for the effectiveness of ongoing performance, directing less attention to external feedback (Fischer \& Ullsperger, 2013; Gehring \& Willoughby, 2002; Nieuwenhuis et al., 2004; Picton, 1992; Polich, 2007; Yeung et al., 2005). 
These results agree with our proposal that individuals whose performance rely mostly on internal-rule-based knowledge (i.e., High ET) are less responsive to and less dependent on external environmental information, operating predominately in a feedback-independent mode.

It is important to mention that the observed differences in EEG activity between the two groups cannot be entirely explained by group differences on the general processing of external feedback information. The differences between the two groups were diminished during the anticipation (SPN component) and evaluation (P3 component) of Final reversal errors (i.e., error feedback that was followed by correct rulebased behavioral switches; see Figs. 3 and 5). This result suggests that despite the fact that individuals with different degrees of ET show different responsiveness to spurious error feedback, once they have collected enough internal evidence that task contingencies have actually changed, their neural patterns of EEG activity predicting behavioral changes converge to a common path.

The observed SPN increase over centro-parietal regions prior to Final reversal errors (see Fig. 4A) is in line with previous findings showing an augmented amplitude of this component prior to the presentation of motivationally relevant information (Brunia et al., 2011; Fuentemilla et al., 2013; Kotani et al, 2003; Moris et al., 2013). The absence of group differences on the SPN amplitude during this period points to similar attention orientation processes toward cues that are perceived as more salient and relevant to behavioral adjustments (Fuentemilla et al., 2013). Furthermore, the increase of the centro-parietal P3 component during final reversal errors converges with recent findings, suggesting that this neural signal is involved in the accumulation of internal evidences leading to the awareness/detection of relevant events requiring the formation of new behaviors (Fischer \& Ullsperger, 2013; O'Connell, Dockree, \& Kelly, 2012), as, for instance, the awareness that an error has been made (Steinhauser \& Yeung, 2010) and behavioral changes are required. Thus, similar increases of SPN and P3 activity during Final reversal errors, in both groups, suggests that even though individuals with High and Low ET display different degrees of attention, motivation, and generate different expectations toward spurious external feedbacks, they share common adaptive cortical mechanisms of updating error values when becoming aware that erratic trends in action require new behavioral solutions.

One interesting framework that fits well with the present findings is the adaptive gain theory of the locus-coeruleus norepinephrine (LC-NE) system of Aston-Jones and Cohen (2005). This theory holds that the LC-NE system is potentially involved in modulating information processing by increasing the signal-to-noise ratio of relevant information (ServanSchreiber, Rajkowski, \& Aston-Jones, 1999). Accordingly, phasic LC-NE activation is driven by the relevance of task- related feedback information according to the task goals. Based on this theory it has been suggested that P3 enhancements might reflect a phasic increase in the response of neocortical neuronal assemblies to the detection of a motivationally salient event (Nieuwenhuis, Aston-Jones, \& Cohen, 2005), independent of its emotional valence (Berridge \& Waterhouse, 2003). Considering this hypothesis, the differences in P3 activity between the two groups might expose general differences in NE-induced phasic responses to external feedback which are related with distinct selective attention and detection thresholds to incoming sensory signals. Furthermore, it has also been shown that administration of yohimbine, which stimulates firing in the LC-NE release and enhances the activity of error-related ERP activity in RT tasks, specifically the ERN component (Riba, Rodriguez-Fornells, Morte, Münte, \& Barbanoj, 2005). As the FRN and the ERN reflect similar brain computations involved in error evaluation (Holroyd \& Coles, 2002), the observed differences between the two groups regarding the FRN signal (see Fig. 3) might as well provide further evidences of the involvement of the LC-NE system in the modulation of ET. An interesting hypothesis to explore is to which extent the amplitude of the SPN component might be related to the LC-NE system.

One of the possible limitations of the present study is that we used extreme groups of ET defined according their performance in the reversal learning task. Interestingly, further studies should be addressed considering participants with moderated patterns of ET as well as using a continuum of ET scores rather than splitting individuals into extreme groups. However, it is worth mentioning that the definition of ET in the present study is driven by the observed performance in a task involving rule-based decisions. Despite the present limitation, we believe that the present study provides interesting neurophysiological evidences supporting discrete cognitive and motivational brain states underlying human differences in ET during the processing of error feedback cues. Moreover, this study not only contributes to a better understanding of the mental processes underlying different type of mistakes performed by human operators in error-prone environments but also point to the importance of taking into account individual differences related to the monitoring of complex chains of commands (Norman, 1988; Reason, 1990) when examining and judging different mistakes performed by human operators in their interaction with real-working environments.

Acknowledgments We wish to thank J. Marco Pallares for his help during the initial stages of this project, and Ernest Mas-Herrero and Joaquin Moris for their comments to previous versions of this article. This research has been supported by a grant from the Spanish Government (PSI2011-29219 to A.R.F.) and the Catalan Government (Generalitat de Catalunya, 2009 SGR 93). 


\section{References}

Albert, R., Jeong, H., \& Barabasi, A. -L. (2000). Error and atack tolerance of complex networks. Nature, 406, 378-382.

Aston-Jones, G., \& Cohen, J. (2005). An integrative theory of locus coeruleus-norepinephrine function: Adaptive gain and optimal performance. Annual Review of Neuroscience, 28, 403-450.

Bandura, A. (1989). Human agency in social cognitive theory. American Psychologist, 44, 1175-1184.

Barcelo, F., Escera, C., Corral, M. J., \& Perianez, J. A. (2006). Task switching and novelty processing activate a common neural network for cognitive control. Journal of Cognitive Neuroscience, 18, 1734 1748.

Berridge, C. W., \& Waterhouse, B. D. (2003). The locus coeruleusnoradrenergic system: Modulation of behavioral state and statedependent cognitive processes. Brain Research Reviews, 42, 33-84.

Botvinick, M. M., Braver, T. S., Barch, D. M., Carter, C. S., \& Cohen, J. D. (2001). Conflict monitoring and cognitive control. Psychological Review, 108, 624-652.

Brunia, C. H., Hackley, S. A., van Boxtel, G. J., Kotani, Y., \& Ohgami, Y. (2011). Waiting to perceive: Reward or punishment? Clinical Neurophysiology, 122, 858-868.

Cañas, J. J., Quesada, J. F., Antoli, A., \& Fajardo, I. (2003). Cognitive flexibility and adaptability to environmental changes in dynamic complex problem-solving tasks. Ergonomics, 46, 482-501.

Carter, C., Braver, T., Barch, D., Botvinick, M., Noll, D., \& Cohen, J. (1998). Anterior cingulate cortex, error detection, and the online monitoring of performance. Science, 280, 747-749.

Carver, C. S., \& White, T. L. (1994). Behavioral inhibition, behavioral activation, and affective responses to impending reward and punishment: The BIS/BAS scales. Journal of Personality and Social Psychology, 67, 319-333.

Casey, S. M. (2006). The atomic chef: And other true tales of design, technology, and human error. Santa Barbara, CA: Aegean.

Chase, H. W., Swainson, R., Durham, L., Benham, L., \& Cools, R. (2011). Feedback-related negativity codes prediction error but not behavioral adjustment during probabilistic reversal learning. Journal of Cognitive Neuroscience, 23, 936-946.

Cunillera, T., Fuentemilla, Ll., Periañez, J., Marco-Pallarès, J., Krämer, U., Càmara, E., ... Rodríguez-Fornells, A. (2012). Brain oscillatory activity associated to task-switching and feedback processing. Cognitive, Affective, and Behavioral Neuroscience, 12, 16-33.

Donchin, E., \& Coles, M. G. H. (1988). Is the P300 component a manifestation of context updating? Behavioral and Brain Sciences, 11, 357-374.

Fields, R. E., Wright, P. C., \& Harrison, M. D. (1995). A task centered approach to analysing human error tolerance requirements. In (pp. 18-26). doi:10.1109/ISRE.1995.512542.

Fischer, A. G., \& Ullsperger, M. (2013). Real and fictive outcomes are processed differently but converge on a common adaptive mechanism. Neuron, 79, 1243-1255.

Fuentemilla, L., Cucurell, D., Marco-Pallares, J., Guitart-Masip, M., Moris, J., \& Rodriguez-Fornells, A. (2013). Electrophysiological correlates of anticipating improbable but desired events. NeuroImage, 78, 135-144.

Gehring, W. J., Goss, B., Coles, M. G. H., Meyer, D. E., \& Donchin, E. (1993). A neural system for error detection and compensation. Psychological Science, 4, 385-390.

Gehring, W. J., \& Willoughby, A. R. (2002). The medial frontal cortex and the rapid processing of monetary gains and losses. Science, 295, 2279-2282.

Gratton, G., Coles, M. G. H., Sirebaag, E. G., Eriksen, C. W., \& Donchin, E. (1988). Pre- and poststimulus activation of response channels: A psychophysiological analysis. Journal of Experimental Psychology: Human Perception and Performance, 14, 331-344.
Holroyd, C. B., \& Coles, M. G. (2002). The neural basis of human error processing: Reinforcement learning, dopamine, and the error-related negativity. Psychological Review, 109, 679-709.

Houde, J. F., \& Nagarajan, S. S. (2011). Speech production as state feedback control. Frontiers in Human Neuroscience, 5. doi:10.3389/ fnhum.2011.00082.

Jeong, H., Tombor, B., Albert, R., Oltvai, Z., \& Barabasi, A. -L. (2001). The large-scale organization of metabolic network. Nature, 407, 654.

Jocham, G., Neumann, J., Klein, T. A., Danielmeier, C., \& Ullsperger, M. (2009). Adaptive coding of action values in the human rostral cingulate zone. Journal of Neuroscience, 29, 7489-7496.

Kotani, Y., Kishida, S., Hiraku, S., Suda, K., Ishii, M., \& Aihara, Y. (2003). Effects of information and reward on stimulus-preceding negativity prior to feedback stimuli. Psychophysiology, 40, 818 826.

Marco-Pallares, J., Camara, E., Munte, T. F., \& Rodriguez-Fornells, A. (2008). Neural mechanisms underlying adaptive actions after slips. Journal of Cognitive Neuroscience, 20, 1595-1610.

Masaki, H., Takeuchi, S., Gehring, W. J., Takasawa, N., \& Yamasaki, K. (2006). Affective-motivational influences on feedback-related ERPs in a gambling task. Brain Research, 1105, 110-121.

Moris, J., Luque, D., \& Rodriguez-Fornells, A. (2013). Learning-induced modulations of the stimulus-preceding negativity. Psychophysiology, 50, 931-939.

Nieuwenhuis, S., Aston-Jones, G., \& Cohen, J. (2005). Decision making, the p3, and the locus coeruleus-norepinephrine system. Psychological Review, 131, 510-532.

Nieuwenhuis, S., Holroyd, C. B., Mol, N., \& Coles, M. G. H. (2004). Reinforcement-related brain potentials from medial frontal cortex: Origins and functional significance. Neuroscience and Biobehavioral Reviews, 28, 441-448.

Norman, D. A. (1981). Categorization of action slips. Psychological Review, 88, 1-15.

Norman, D. A. (1988). The design of everyday things. New York, NY: Doubleday.

Norman, D. A., \& Shallice, T. (1986). Attention to action: Willed and automatic control of behavior. In R. J. Davidson, D. E. Schwartz, \& D. Shapiro (Eds.), Consciousness and self-regulation (pp. 1-18). New York, NY: Plenum Press.

O’Connell, R. G., Dockree, P. M., \& Kelly, S. P. (2012). A supramodal accumulation-to-bound signal that determines perceptual decisions in humans. Nature Neuroscience, 15, 1729-1735.

Picton, T. W. (1992). The P300 wave of the human event-related potential. Journal of Clinical Neurophysiology, 9, 456-479.

Polich, J. (2007). Updating P300: An integrative theory of P3a and P3b. Clinical Neurophysiology, 118, 2128-2148.

Rasmussen, J. (1983). Skills, rules and knowledge: Signals, signs and symbols distinctions in human performance models. IEEE Transactions on Systems, Man and Cybernetics, SMC-13, 257-266.

Rasmussen, J., \& Vicente, K. J. (1989). Coping with human errors through system design: implications from ecological interface design. International Journal of Man-Machine Studies, 31, 517-534.

Reason, J. (1990). Human error. Cambridge, UK: Cambridge University Press.

Riba, J., Rodriguez-Fornells, A., Morte, A., Münte, T. F., \& Barbanoj, M. J. (2005). Noradrenergic stimulation enhances human action monitoring as revealed by event-related brain potentials. Journal of Neuroscience, 25, 4370-4374.

Ridderinkhof, K. R., Ramautar, J. R., \& Wijnen, J. G. (2009). To PE or not to PE: A P3-like ERP component reflecting the processing of response errors. Psychophysiology, 46, 531-538.

Ridderinkhof, K. R., Ullsperger, M., Crone, E. A., \& Nieuwenhuis, S. (2004). The role of the medial frontal cortex in cognitive control. Science, 306, 443-447. 
Rodriguez-Fornells, A., Kurzbuch, A. R., \& Munte, T. F. (2002). Time course of error detection and correction in humans: Neurophysiological evidence. Journal of Neuroscience, 22, 99909996.

Rougier, N. P., Noelle, D. C., Braver, T. S., Cohen, J. D., \& O’Relly, R. C. (2005). Prefrontal cortex and flexible cognitive control: Rules without symbols. PNAS, 12, 7338-7343.

Servan-Schreiber, D., Rajkowski, J., \& Aston-Jones, G. (1999). The role of locus coeruleus in the regulation of cognitive performance. Science, 283, 549-554.

Spiro, R. J., Coulson, R. L., Feltovich, P. J., \& Andersib, D. K. (1988). Cognitive flexibility theory: Advanced knowledge acquisition in illstructured domains. In V. Patel (Ed.), Proceedings of the 10th annual conference of the Cognitive Science Society. Hillsdale, NJ: Erlbaum.

Steinhauser, M., \& Yeung, N. (2010). Decision processes in human performance monitoring. Journal of Neuroscience, 30, 15643-15653.

Sternad, D., Abe, M. O., Hu, X., \& Muller, H. (2011). Neuromotor noise, error tolerance and velocitydependent costs in skilled performance. PLoS Comput Biol, 7, e1002159. doi:10.1371/journal.pcbi.1002159.
Torrubia, R., Ávila, C., Moltó, J., \& Caseras, X. (2001). The Sensitivity to Punishment and Sensitivity to Reward Questionnaire (SPSRQ) as a measure of Gray's anxiety and impulsivity dimensions. Personality and Individual Differences, 31, 837-862.

Ullsperger, M., Danielmeier, C., \& Jocham, G. (2014a). Neurophysiology of performance monitoring and adaptive behavior. Physiological Reviews, 94, 35-79.

Ullsperger, M., Fischer, A. G., Nigburg, R., \& Endrass, T. (2014b). Neural mechanisms and temporal dynamics of performance monitoring. Trends in Cognitive Science, 18, 259-267.

Wiegmann, D. A., \& Shappell, S. A. (2003). A human error approach to aviation accident analysis. Aldershot, UK: Ashgate.

Woods, D. D. (1994). Behind human error: Cognitive systems, computers, and hindsight. Wright-Patterson Air Force Base, OH: Crew System Ergonomics Information Analysis Center.

Yeung, N., Holroyd, C. B., \& Cohen, J. D. (2005). ERP correlates of feedback and reward processing in the presence and absence of response choice. Cerebral Cortex, 15, 535-544. 